\title{
A DECOMPOSITION THEOREM FOR SUBMEASURES
}

\author{
by A. R. KHAN and K. ROWLANDS
}

(Received 11 October, 1983)

1. Introduction. In recent years versions of the Lebesgue and the Hewitt-Yosida decomposition theorems have been proved for group-valued measures. For example, Traynor [4], [6] has established Lebesgue decomposition theorems for exhaustive groupvalued measures on a ring using (1) algebraic and (2) topological notions of continuity and singularity, and generalizations of the Hewitt-Yosida theorem have been given by Drewnowski [2], Traynor [5] and Khurana [3]. In this paper we consider group-valued submeasures and in particular we have established a decomposition theorem from which analogues of the Lebesgue and Hewitt-Yosida decomposition theorems for submeasures may be derived. Our methods are based on those used by Drewnowski in [2] and the main theorem established generalizes Theorem 4.1 of [2].

2. Notation and terminology. Let $G$ be a commutative lattice group (abbreviated to $l$-group). A quasi-norm (resp. norm) $q$ on $G$ is said to be an $l$-quasi-norm (l-norm) if $q(x) \leqslant q(y)$ for all $x, y$ in $G$ with $|x| \leqslant|y|$. A $G$-valued function $\mu$ defined on a ring $\mathscr{R}$ of subsets of a set $X$ is said to be a submeasure if $\mu(\varnothing)=0, \mu(E \cup F) \leqslant \mu(E)+\mu(F)$ for all $E, F$ in $\mathscr{R}$ with $E \cap F=\varnothing$, and $\mu(E) \leqslant(F)$ for all $E, F$ in $\mathscr{R}$ with $E \subseteq F$. A $G$-valued submeasure $\mu$ on $\mathscr{R}$ is said to be exhaustive if and only if, for any disjoint sequence $\left\{E_{n}\right\}$ in $\mathscr{R}, \lim _{n \rightarrow \infty} \mu\left(E_{n}\right)=0$ in $(G, q)$. An l-group $G$ is said to be order complete if every bounded increasing net in $G$ has a supremum. An $l$-quasi-norm $q$ on $G$ is said to be order continuous if $\varnothing \subset A \uparrow x$ in $G^{+}=\{x \in G: x \geqslant 0\}$ implies $q(x)=\sup \{q(y): y \in A\}$ and $B \downarrow x$ in $G^{+}$implies $q(x)=\inf \{q(y): y \in B\}$.

Let $\mathscr{D}$ denote a collection of pairwise disjoint sets in $\mathscr{R}$ and let $\Delta$ be the set of all such collections. If $\mathscr{D}_{1}, \mathscr{D}_{2} \in \Delta$, then we write $\mathscr{D}_{1} \leqslant \mathscr{D}_{2}$ if and only if $\mathscr{D}_{2}$ is a refinement of $\mathscr{D}_{1}$. With each $E \in \mathscr{R}$ we associate members of $\mathscr{D}$; the collection of all such pairs $(E, \mathscr{D})$ is denoted by $\mathscr{G}$ and we let

$$
\mathscr{G}(E)=\{\mathscr{D} \in \Delta:(E, \mathscr{D}) \in \mathscr{G}\} \quad \text { and } \quad \Delta_{\mathscr{G}}=\bigcup_{E \in \mathscr{R}} \mathscr{G}(E)
$$

In the sequel we use $\bigcup \mathscr{D}$ to mean the set theoretic union of the members of $\mathscr{D}$. Following Drewnowski's terminology ([2], Definition 2.1), the collection $\mathscr{G}$ is said to be an additivity on $\mathscr{R}$ if it satisfies the following conditions:

(a) $\Delta_{f} \subseteq \Delta_{g}$, where $\Delta_{f}$ consists of those collections $\mathscr{D}$ which have only a finite number of members;

(b) if $E \in \mathscr{R}$ and $\mathscr{D} \in \mathscr{G}(E)$, then $\bigcup \mathscr{D}=E$;

(c) if $E \in \mathscr{R}, \mathscr{D}_{1}, \mathscr{D}_{2} \in \mathscr{G}(E)$, then $\mathscr{D}_{1} \cap \mathscr{D}_{2} \in \mathscr{G}(E)$, where $\mathscr{D}_{1} \cap \mathscr{D}_{2}=\left\{D_{1} \cap D_{2}: D_{i} \in \mathscr{D}_{i}\right.$, $i=1,2\}$.

Glasgow Math. J. 26 (1985) 69-74. 
(d) if $E_{1}, E_{2} \in \mathscr{R}, E_{1} \cap E_{2}=\varnothing$ and $\mathscr{D}_{i} \in \mathscr{G}\left(E_{i}\right)(i=1,2)$, then $\mathscr{D}_{1} \cup \mathscr{D}_{2} \in \mathscr{G}\left(E_{1} \cup E_{2}\right)$, where $\mathscr{D}_{1} \cup \mathscr{D}_{2}=\left\{D_{1} \cup D_{2}: D_{i} \in \mathscr{D}_{i}, i=1,2\right\}$;

(e) if $E, F \in \mathscr{R}, E \subseteq F$ and $\mathscr{D} \in \mathscr{G}(F)$, then $\mathscr{D} \cap E \in \mathscr{G}(E)$.

Examples of additivities are

1. $\mathscr{G}_{f}=\left\{(E, \mathscr{D}): E \in \mathscr{R}, \mathscr{D} \in \Delta_{f}, \bigcup \mathscr{D}=E\right\}$

2. $\mathscr{G}_{c}=\left\{(E, \mathscr{D}): E \in \mathscr{R}, \mathscr{D} \in \Delta_{c}, \cup \mathscr{D}=E\right\}$, where $\Delta_{c}$ is the collection of all $\mathscr{D}$ which contain a countable number of disjoint sets in $\mathscr{R}$.

A topology $\tau$ on $\mathscr{R}$ is said to be a ring topology if the mappings $(A, B) \rightarrow A \Delta B$ and $(A, B) \rightarrow A \cap B$ of $\mathscr{R} \times \mathscr{R} \rightarrow \mathscr{R}$ are continuous, continuity being with respect to the product topology on $\mathscr{R} \times \mathscr{R}$. A ring topology $\tau$ is said to be an FN-topology (FréchetNikodym) if and only if, for each $\tau$-neighbourhood $U$ of $\varnothing$ in $\mathscr{R}$, there exists a $\tau$-neighbourhood $V$ of $\varnothing$ in $\mathscr{R}$ such that $B \in U$ for all $B \subseteq A \in V, B \in \mathscr{R}$. The notion of an FN-topology was introduced and studied by Drewnowski in ([1], pp. 271-5). In particular, a family $\mathscr{F}=\left\{\eta_{i}: i \in I\right\}$ of $\mathbb{R}_{+}^{*}$-valued submeasures on a ring defines an FN-topology $\Gamma\left(\eta_{i}: i \in I\right)$; a base of $\Gamma\left(\eta_{i}: i \in I\right)$-neighbourhoods of $\varnothing$ in $\mathscr{R}$ being given by finite intersections of sets of the form $U_{\varepsilon, i}=\left\{A \in \mathscr{R}: \eta_{i}(A)<\varepsilon\right\}\left(\varepsilon>0, \eta_{i} \in \mathscr{F}\right)$. Conversely, for each FN-topology $\Gamma$ on $\mathscr{R}$, there is a family $\left\{\xi_{j}: j \in J\right\}$ of $\mathbb{R}_{+}^{*}$-submeasures on $\mathscr{R}$ such that $\Gamma=\Gamma\left(\xi_{j}: J \in J\right)$.

Let $f(\mathscr{D})$ denote finite collections of members of $\mathscr{D}$. If $\Gamma$ is an FN-topology on $\mathscr{R}$ and $E \in \mathscr{R}$, we say that $E=\Gamma-\lim f(\mathscr{D})$ if and only if, for each $\Gamma$-neighbourhood $U$ of $\varnothing$ in $\mathscr{R}$, there exists a $\mathscr{D}_{U} \in f(\mathscr{D})$ such that $E \Delta \cup \mathscr{D}^{\prime} \in U$ for all $\mathscr{D}_{U} \subseteq \mathscr{D}^{\prime} \in f(\mathscr{D})$. We shall also use the following example of an additivity.

3. $\mathscr{G}_{c}(\Gamma)=\left\{(E, \mathscr{D}): E \in \mathscr{R}, \mathscr{D} \in \Delta_{c}, \bigcup \mathscr{D}=E, E=\Gamma\right.$-lim $\left.f(\mathscr{D})\right\}$. The above additivity is called the additivity generated by $\Gamma$. In particular, if $\eta$ is an $\mathbb{R}_{+}^{*}$-valued submeasure on $\mathscr{R}$ we abbreviate $\mathscr{G}_{c}(\Gamma(\eta))$ to $\mathscr{G}(\eta)$; in this case we note that, if $E \in \mathscr{R}$ and $\mathscr{D}=$ $\left\{D_{n}: n=1,2, \ldots\right\} \in \Delta_{c}$, then $E=\eta-\lim f(\mathscr{D})$ if and only if

$$
\eta\left(E \backslash \bigcup_{k=1}^{n} D_{k}\right) \rightarrow 0 \quad \text { as } \quad n \rightarrow \infty .
$$

In proving our decomposition theorem we require the notions of $\mathscr{G}$-continuity and $\mathscr{G}$-singularity as given by Drewnowski in [2], Definitions 2.4 and 2.17 respectively. For the sake of completeness we include these definitions as follows.

DefintTION 1. Let $\mathscr{G}$ be an additivity on $\mathscr{R}$. An FN-topology $\Gamma$ on $\mathscr{R}$ is said to be

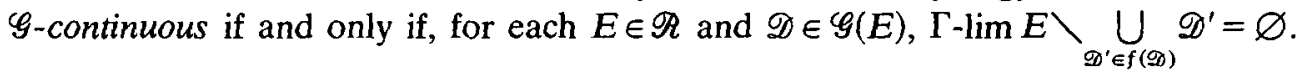

DefintTion 2. An FN-topology $\Gamma$ is said to be $\mathscr{G}$-singular if and only if the only $\mathscr{G}$-continuous FN-topology weaker than $\Gamma$ is the trivial one.

If $(G, q)$ is an $l$-quasi-normed group and $\eta$ is a $G$-valued submeasure on $\mathscr{R}$, then clearly $\Gamma\left(q^{\circ} \eta\right)$ is $\mathscr{G}(\eta)$-continuous. We also see that, if $\mathscr{G}$ is an additivity on $\mathscr{R}$, then $\Gamma\left(q^{\circ} \eta\right)$ is $\mathscr{G}$-continuous if and only if, for each $E \in \mathscr{R}$ and $\mathscr{D} \in \mathscr{G}(E)$, $\lim _{\mathscr{D} \in f(\mathscr{D})} q\left(\eta\left(E \backslash \cup \mathscr{D}^{\prime}\right)\right)=0$; in this case we simply say that $\eta$ is $\mathscr{G}$-continuous. It is also 
straightforward to show that an FN-topology $\Gamma$ is $\mathscr{G}_{c}$-continuous if and only if it is order continuous; that is, if $\left\{E_{n}: n=1,2, \ldots\right\}$ is a sequence in $\mathscr{R}, E_{n} \downarrow \varnothing$, then $\Gamma$ - $\lim _{n} E_{n}=\varnothing$. In a similar way we say that $\eta$ is $\mathscr{G}$-singular if and only if $\Gamma(q \circ \eta)$ is $\mathscr{G}$-singular. It is not difficult to prove that $\eta$ is $\mathscr{G}$-singular if and only if any $\mathscr{G}$-continuous $G$-valued submeasure $\lambda$ on $\mathscr{R}$ such that $\lambda \ll \eta$ is identically zero.

3. The decomposition theorem. In this section we assume that $G$ is an order complete $l$-group and that $q$ is an order continuous $l$-quasi-norm on $G$. Let $\mu$ be an exhaustive $G$-valued submeasure on $\mathscr{R}$ and suppose that $\mathscr{G}$ is an additivity on $\mathscr{R}$. For each $E \in \mathscr{R}$, define

and

$$
S_{\mu}(E)=\bigwedge_{\mathscr{D} \in \mathcal{G}(E)} \bigvee_{\mathscr{D}^{\prime} \in f(\mathscr{D})} \mu\left(\bigcup \mathscr{D}^{\prime}\right)
$$

$$
S_{\mu}^{\prime}(E)=\bigvee_{\mathscr{D} \in \mathscr{G}(E)} \bigwedge_{\mathscr{D}^{\prime} \in f(\mathscr{D})} \mu\left(E \backslash \bigcup \mathscr{D}^{\prime}\right)
$$

Then we have the following

Lemma 1. $S_{\mu}$ and $S_{\mu}^{\prime}$ are $G$-valued exhaustive submeasures on $\mathscr{R}$.

Proof. Let $E \in \mathscr{R}$ and $\mathscr{D} \in \mathscr{G}(E)$. By property (b) of an additivity $\bigcup \mathscr{D}=E$ and so $0 \leqslant \mu\left(\bigcup \mathscr{D}^{\prime}\right) \leqslant \mu(E)$ for all $\mathscr{D}^{\prime} \in f(\mathscr{D})$; the net $\left\{\mu\left(\bigcup \mathscr{D}^{\prime}\right): \mathscr{D}^{\prime} \in f(\mathscr{D})\right\}$ is $\uparrow$ and bounded and so by the order completeness of $G \underset{\mathscr{D} \prime \in f(\mathscr{D})}{\vee} \mu\left(\bigcup \mathscr{D}^{\prime}\right)$ exists. Similarly, by property (c) of an additivity the net $\left\{\bigvee_{\mathscr{D} \in f(\mathscr{D})} \mu\left(\bigcup \mathscr{D}^{\prime}\right): \mathscr{D} \in \mathscr{G}(E)\right\}$ is $\downarrow$ and bounded and so by the order completeness of $G \bigwedge_{\mathscr{D} \in \mathscr{G}(E)} \bigvee_{\mathscr{D}^{\prime} \in f(\mathscr{D})} \mu\left(\cup \mathscr{D}^{\prime}\right)$ exists in $G^{+}$for each $E \in \mathscr{R}$. By a similar argument we can prove that $S_{\mu}^{\prime}(E)$ exists in $G^{+}$for each $E \in \mathscr{R}$.

The subadditivity of $S_{\mu}$ (resp. $S_{\mu}^{\prime}$ ) follows from the subadditivity of $\mu$ and property (d) (property (e)) of an additivity. Similarly the monotonicity of $S_{\mu}$ (resp. $S_{\mu}^{\prime}$ ) follows from the monotonicity of $\mu$ and property (c) (resp. (d)) of an additivity.

For any $E \in \mathscr{R}, S_{\mu}(E) \leqslant \mu(E)$ and $S_{\mu}^{\prime}(E) \leqslant \mu(E)$, and so, since $q$ is an l-quasi-norm, $q\left(S_{\mu}(E)\right) \leqslant q(\mu(E))$ and $q\left(S_{\mu}^{\prime}(E)\right) \leqslant q(\mu(E))$; this implies that both $S_{\mu}$ and $S_{\mu}^{\prime}$ are exhaustive and $\mu$-continuous.

LEMMA 2. (i) $S_{\mu}$ is $\mathscr{G}$-continuous.

(ii) $S_{\mu}^{\prime}$ is $\mathscr{G}$-singular.

Proof. (i) Suppose that $S_{\mu}$ is not $\mathscr{G}$-continuous. Then there exist a positive number $\varepsilon$, $E \in \mathscr{R}$ and $\mathscr{D} \in \mathscr{G}(E)$ such that $q\left(S_{\mu}\left(E \backslash \cup \mathscr{D}^{\prime}\right)\right)>\varepsilon$ for all $\mathscr{D}^{\prime} \in f(\mathscr{D})$. Since $S_{\mu}$ is a submeasure and $q$ has the $l$-property we have

$$
q\left(S_{\mu}(E)\right) \geqslant q\left(S_{\mu}\left(E \backslash \bigcup \mathscr{D}^{\prime}\right)\right)>\varepsilon
$$

for all $\mathscr{D}^{\prime} \in f(\mathscr{D})$; also, $S_{\mu}(E) \leqslant \bigvee_{\mathscr{D}^{\prime} \in f(\mathscr{D})} \mu\left(\bigcup \mathscr{D}^{\prime}\right)$ and since $q$ is order continuous 
$\sup _{\mathscr{D}^{\prime} \in f(\mathscr{D})} q\left(\mu\left(\bigcup \mathscr{D}^{\prime}\right)\right) \geqslant q\left(S_{\mu}(E)\right)>\varepsilon$. Thus there exists a $\mathscr{D}_{1} \in f(\mathscr{D})$ such that $q\left(\mu\left(\bigcup \mathscr{D}_{1}\right)\right)>\varepsilon$. By property (e) of an additivity $\mathscr{D} \backslash \mathscr{D}_{1} \in \mathscr{G}\left(E \backslash \cup \mathscr{D}_{1}\right)$, where $\mathscr{D} \backslash \mathscr{D}_{1}=\left\{D \in \mathscr{D}: D \notin \mathscr{D}_{1}\right\}$, and from (1) $q\left(S_{\mu}\left(E \backslash \cup \mathscr{D}_{1}\right)\right)>\varepsilon$. It follows from the order continuity of $q$ that $\sup q\left(\mu\left(\bigcup \mathscr{D}^{\prime}\right)\right) \geqslant q\left(S_{\mu}\left(E \backslash \bigcup \mathscr{D}_{1}\right)\right)>\varepsilon$ and so there exists a $\mathscr{D}_{2} \in f\left(\mathscr{D} \backslash \mathscr{D}_{1}\right)$ such that $\mathscr{D}^{\prime} \in \mathbf{f}\left(\mathscr{D} \backslash \mathscr{D}_{1}\right)$ $q\left(\mu\left(\bigcup \mathscr{D}_{2}\right)\right)>\varepsilon$

In this way we construct by induction a disjoint sequence $\left\{\mathscr{D}_{n}: n=1,2, \ldots\right\}$ such that $q\left(\mu\left(\bigcup \mathscr{D}_{n}\right)\right)>\varepsilon$. This contradicts the exhaustive property of $\mu$, and so $\mu$ is $\mathscr{G}$-continuous, as required.

(ii) Suppose that $S_{\mu}^{\prime}$ is not $\mathscr{G}$-singular. Then there exists a $\mathscr{G}$-continuous $G$-valued submeasure $\lambda$ such that $\lambda \ll S_{\mu}^{\prime}$ and $\lambda$ is not identically zero. This implies that there is a set $E \in \mathscr{R}$ and a positive number $\eta$ such that $q(\lambda(E))>\eta>0$. Since $\lambda \ll S_{\mu}^{\prime}$ there is a positive number $\delta$ such that

$$
q\left(S_{\mu}^{\prime}(F)\right)<\delta \Rightarrow q(\lambda(F))<\eta / 2 \quad(F \in \mathscr{R}) .
$$

Thus $q\left(S_{\mu}^{\prime}(E)\right) \geqslant \delta$; since $q$ is order continuous there exists a $\mathscr{D} \in \mathscr{G}(E)$ such that $q\left(\mu\left(E \backslash \bigcup \mathscr{D}^{\prime}\right)\right) \geqslant \delta$ for all $\mathscr{D}^{\prime} \in f(\mathscr{D})$. Now $\lambda$ is $\mathscr{G}$-continuous and so there exists a $\mathscr{D}_{0}^{\prime} \in f(\mathscr{D})$ such that $q\left(\lambda\left(E \backslash \bigcup \mathscr{D}_{0}^{\prime}\right)\right)<\eta / 2^{2}$. Let $E_{1}=\bigcup \mathscr{D}_{0}^{\prime}$ and $A_{1}=E \backslash E_{1}$. Then $q\left(\mu\left(A_{1}\right)\right) \geqslant \delta, q\left(\lambda\left(A_{1}\right)\right)<\eta / 2^{2}$ and $q\left(\lambda\left(E_{1}\right)\right)>\eta / 2+\eta / 2^{2}$. Thus from $(2) q\left(S_{\mu}^{\prime}\left(E_{1}\right)\right) \geqslant \delta$ and so there exists a $\mathscr{D} \in \mathscr{G}\left(E_{1}\right)$ such that $q\left(\mu\left(E_{1} \backslash \bigcup \mathscr{D}^{\prime}\right)\right) \geqslant \delta$ for all $\mathscr{D}^{\prime} \in f(\mathscr{D})$. Again since $\lambda$ is $\mathscr{G}$-continuous there exists a $\mathscr{D}_{1}^{\prime} \in f(\mathscr{D})$ such that $q\left(\lambda\left(E_{1} \backslash \bigcup \mathscr{D}_{1}^{\prime}\right)\right)<\eta / 2^{3}$. Let $E_{2}=\bigcup \mathscr{D}_{1}^{\prime}$ and $A_{2}=E_{1} \backslash E_{2}$. Then $q\left(\mu\left(A_{2}\right)\right) \geqslant \delta, q\left(\lambda\left(A_{2}\right)\right)<\eta / 2^{3}$ and $q\left(\lambda\left(E_{2}\right)\right)>\eta / 2+\eta / 2^{3}$. In this way we construct by induction a disjoint sequence $\left\{A_{n}: n=1,2, \ldots\right\}$ in $\mathscr{R}$ such that $q\left(\mu\left(A_{n}\right)\right) \geqslant \delta$ for $n=1,2, \ldots$. This contradicts the property that $\mu$ is exhaustive.

LEMMA 3. (i) If $\lambda$ is a $\mathscr{G}$-continuous $G$-valued submeasure on $\mathscr{R}$ such that $\lambda \ll \mu$, then $\lambda \ll S_{\mu}$.

(ii) If $\nu$ is a $\mathscr{G}$-singular $G$-valued submeasure on $\mathscr{R}$ such that $\nu \ll \mu$, then $\nu \ll S_{\mu}^{\prime}$.

Proof. (i) Since $\lambda \ll \mu$, given any $\varepsilon>0$, there exists a positive $\delta$ such that

$$
q(\mu(E))<\delta \Rightarrow q(\lambda(E) \leqslant \varepsilon \quad(E \in R) .
$$

We seek to show that $q\left(S_{\mu}(E)\right)<\delta \Rightarrow q(\lambda(E)) \leqslant \varepsilon$. Suppose that this assertion is not true. Then there exists an $E_{0}$ in $\mathscr{R}$ such that $q\left(S_{\mu}\left(E_{0}\right)\right)<\delta$ and $q\left(\lambda\left(E_{0}\right)\right)>\varepsilon+\gamma$ for some positive number $\gamma$. Since $q$ is order continuous there exists $\mathscr{D} \in \mathscr{G}\left(E_{0}\right)$ such that $q\left(\mu\left(\bigcup \mathscr{D}^{\prime}\right)\right)<\delta$ for all $\mathscr{D}^{\prime} \in f(\mathscr{D})$. Since $\lambda$ is $\mathscr{G}$-continuous there exists a $\mathscr{D}_{0}^{\prime} \in f(\mathscr{D})$ such that $q\left(\lambda\left(E_{0} \backslash \bigcup \mathscr{D}_{0}^{\prime}\right)\right)<\gamma / 2$. It follows that $q\left(\lambda\left(\bigcup \mathscr{D}_{0}^{\prime}\right)\right)>\varepsilon+\gamma / 2$. Thus $q\left(\mu\left(\bigcup \mathscr{D}_{0}^{\prime}\right)\right)<\delta$ and $q\left(\lambda\left(\bigcup \mathscr{D}_{0}^{\prime}\right)\right)>\varepsilon+\gamma / 2$. This contradicts (3), and so $\lambda \ll S_{\mu}$.

(ii) Since $\nu \ll \mu$, given any $\varepsilon>0$, there exists a positive number $\delta$ such that

$$
q(\mu(E))<\delta \Rightarrow q(\nu(E)) \leqslant \varepsilon \quad(E \in R) .
$$

We seek to prove that $q\left(S_{\mu}^{\prime}(E)\right)<\delta \Rightarrow q(\nu(E)) \leqslant \varepsilon$. Suppose that the implication is not true. Then there exists a set $E_{0}$ in $\mathscr{R}$ such that $q\left(S_{\mu}^{\prime}\left(E_{0}\right)\right)<\delta \Rightarrow q\left(\nu\left(E_{0}\right)\right)>\varepsilon+\gamma$ for some 
$\gamma>0$. This implies that for all $\mathscr{D} \in \mathscr{G}\left(E_{0}\right), q\left(\bigwedge_{\mathscr{D}^{\prime} \in f(\mathscr{D})} \mu\left(E_{0} \backslash \cup \mathscr{D}^{\prime}\right)\right)<\delta$. Since $\nu \ll \mu$ and $\mu$ is exhaustive it follows that $\nu$ is exhaustive and so, by Lemma $2(i), S_{\nu}$ is $\mathscr{G}$-continuous. Moreover, $S_{\nu} \ll \nu$ and so, since $\nu$ is $\mathscr{G}$-singular, it follows that $S_{\nu}=0$. Thus there exists a $\mathscr{D}_{0} \in \mathscr{G}\left(E_{0}\right)$ such that $q\left(\nu\left(\bigcup \mathscr{D}^{\prime}\right)\right)<\gamma / 2$ for all $\mathscr{D}^{\prime} \in f\left(\mathscr{D}_{0}\right)$. Choose $\mathscr{D}_{0}^{\prime} \in f\left(\mathscr{D}_{0}\right)$ so that $q\left(\mu\left(E_{0} \backslash \bigcup \mathscr{D}_{0}^{\prime}\right)\right)<\delta$ and let $F_{0}=\bigcup \mathscr{D}_{0}^{\prime}$. Then $q\left(\nu\left(E_{0} \backslash F_{0}\right)\right)>\varepsilon+\gamma-\gamma / 2=\varepsilon+\gamma / 2$. This contradicts (4) and so $\nu \ll S_{\mu}^{\prime}$.

Defintrion 3. Two $G$-valued submeasures $\mu, \nu$ defined on a ring $\mathscr{R}$ are said to be equivalent, written $\mu \sim \nu$, if and only if $\mu \ll \nu$ and $\nu \ll \mu$.

We now prove our decomposition theorem.

THEOREM 1. Let $(G, q)$ be an l-group and $q$ an order continuous l-norm on $G$. Let $\mu$ be an exhaustive $G$-valued submeasure on $\mathscr{R}$ and $\mathscr{G}$ an additivity on $\mathscr{R}$. Then $\mu \sim S_{\mu}+S_{\mu}^{\prime}$ $\left(\sim S_{\mu} \vee S_{\mu}^{\prime}\right)$. If $\lambda, \nu$ are $\mathscr{G}$-continuous and $\mathscr{G}$-singular $G$-valued submeasures on $\mathscr{R}$ respectively such that $\mu \sim \lambda+\nu$, then $\lambda \sim S_{\mu}$ and $\nu \sim S_{\mu}^{\prime}$.

Proof. Let $E \in \mathscr{R}, \mathscr{D} \in \mathscr{G}(E)$ and $\mathscr{D}^{\prime} \in f(\mathscr{D})$. Now

and so

$$
E=\left(E \backslash \cup \mathscr{D}^{\prime}\right) \cup\left(\bigcup \mathscr{D}^{\prime}\right)
$$

$$
\mu(E) \leqslant \mu\left(E \backslash \bigcup \mathscr{D}^{\prime}\right)+\mu\left(\bigcup \mathscr{D}^{\prime}\right) \leqslant \mu\left(E \backslash \bigcup \mathscr{D}^{\prime}\right)+\underset{\mathscr{D}^{\prime} \in f(\mathscr{D})}{\bigvee} \mu\left(\bigcup \mathscr{D}^{\prime}\right)
$$

it follows that

$$
\mu(E) \leqslant \bigwedge_{\mathscr{D}^{\prime} \in f(\mathscr{D})} \mu\left(E \backslash \cup \mathscr{D}^{\prime}\right)+\bigvee_{\mathscr{D}^{\prime} \in f(\mathscr{D})} \mu\left(\bigcup \mathscr{D}^{\prime}\right)
$$

and subsequently we have

$$
\mu(E) \leqslant \bigvee_{\mathscr{D} \in \mathscr{G}(E)} \bigwedge_{\mathscr{D}^{\prime} \in f(\mathscr{D})} \mu\left(E \backslash \bigcup \mathscr{D}^{\prime}\right)+\bigwedge_{\mathscr{D} \in \mathcal{G}_{(E)}(E)} \bigvee_{\mathscr{D}^{\prime} \in f(\mathscr{D})} \mu\left(\bigcup \mathscr{D}^{\prime}\right)
$$

Thus, for $E \in \mathscr{R}$,

$$
\mu(E) \leqslant S_{\mu}(E)+S_{\mu}^{\prime}(E) .
$$

Moreover, $S_{\mu}(E) \leqslant \mu(E)$ and $S_{\mu}^{\prime}(E) \leqslant \mu(E)$, and so it is clear that $\mu \sim S_{\mu}+S_{\mu}^{\prime}$.

The second part of the theorem deals with the 'uniqueness' of the decomposition.

If $\lambda+\nu \sim \mu$, then $\lambda, \nu \ll \mu$. Thus, by Lemma 3, $\lambda \ll S_{\mu}$ and $\nu \ll S_{\mu}^{\prime}$. Also $\lambda+\nu \sim$ $S_{\mu}+S_{\mu}^{\prime}$, so that, in particular, $S_{\mu} \ll \lambda+\nu$ and $S_{\mu}^{\prime} \ll \lambda+\nu$. The $G$-valued submeasure $\lambda+\nu$ is exhaustive and so by Lemma 3

$$
S_{\mu} \ll S_{\lambda+\nu}=S_{\lambda}+S_{\nu} \quad \text { and } S_{\mu}^{\prime} \ll S_{\lambda+\nu}^{\prime}=S_{\lambda}^{\prime}+S_{\nu}^{\prime} \text {. }
$$

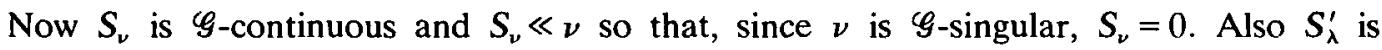
$\mathscr{G}$-singular by Lemma 2 (ii) and since $S_{\lambda}^{\prime} \leqslant \lambda$ and $\lambda$ is $\mathscr{G}$-continuous it follows that $S_{\lambda}^{\prime}$ is $\mathscr{G}$-continuous; thus $S_{\lambda}^{\prime}=0$.

Therefore $S_{\mu} \ll S_{\lambda} \ll \lambda$ and $S_{\mu}^{\prime} \ll S_{\nu}^{\prime} \ll \nu$.

Thus $S_{\mu} \sim \lambda$ and $S_{\mu}^{\prime} \sim \nu$, as required. 
Corollary 1. If $\mathscr{G}=\mathscr{G}_{c}$, then we have a Hewitt-Yosida type decomposition theorem for exhaustive l-group-valued submeasures. In this case $S_{\mu}$ is order continuous and so is a $\sigma$-sub-additive submeasure on $\mathscr{R}$ and $S_{\mu}^{\prime}$ is 'purely finitely sub-additive' in the sense that, if $\lambda$ is an order-continuous $G$-valued submeasure on $\mathscr{R}$ such that $\lambda \ll S_{\mu}^{\prime}$, then $\lambda=0$.

Corollary 2. Let $(E, p)$ be an l-quasi-normed group and let $\eta$ be an E-valued submeasure on $\mathscr{R}$. Suppose that the additivity on $\mathscr{R}$ is $\mathscr{G}=\mathscr{G}_{c}(\Gamma(p \circ \eta))$. In this case we have a Lebesgue-type decomposition theorem for an exhaustive $G$-valued submeasure $\mu$; the submeasure $S_{\mu}$ is $\eta$-continuous and $S_{\mu}^{\prime}$ is $\eta$-singular.

\section{REFERENCES}

1. L. Drewnowski, Topological rings of sets, continuous set functions, integration I, Bull. Acad. Polon. Sci., Sér. Sci. Math.,, Astr. et Phys., 20, (1972), 269-276.

2. L. Drewnowski, Decompositions of set functions, Studia Mathematica 48 (1973), 23-48.

3. S. S. Khurana, Submeasures and decomposition of measures, J. Math. Analysis and Applications 70 (1979), 111-113.

4. T. Traynor, Decomposition of group-valued additive set functions, Ann. Inst. Fourier, Grenoble 22 (1972), 131-140.

5. T. Traynor, A general Hewitt-Yosida decomposition, Canadian J. Math. 24 (1972), 1164-1169.

6. T. Traynor, The Lebesgue decomposition for group-valued set functions, Trans. Amer. Math. Soc. 220 (1976), 307-319.

Department of Mathematics

UNIVERSITY OF MULTAN

PAKISTAN
Department of Pure Mathematics University College of Wales ABERYSTWYTH 